\title{
SYMPLECTIC TORI IN RATIONAL ELLIPTIC SURFACES
}

\author{
TOLGA ETGÜ AND B. DOUG PARK
}

\begin{abstract}
Let $E(1)_{p}$ denote the rational elliptic surface with a single multiple fiber $f_{p}$ of multiplicity $p$. We construct an infinite family of homologous non-isotopic symplectic tori representing the primitive 2-dimensional homology class $\left[f_{p}\right]$ in $E(1)_{p}$ when $p>1$. As a consequence, we get infinitely many non-isotopic symplectic tori in the fiber class of the rational elliptic surface $E(1) \cong \mathbb{C P}^{2} \# 9 \overline{\mathbb{C P}}^{2}$. We also show how these tori can be non-isotopically embedded as homologous symplectic submanifolds in other symplectic 4-manifolds.
\end{abstract}

\section{INTRODUCTION}

This paper is a continuation of studies initiated in [EP1] regarding infinite families of non-isotopic and symplectic tori representing the same homology class in a symplectic 4manifold. Let $E(1) \cong \mathbb{C P}^{2} \# 9 \overline{\mathbb{C P}}^{2}$ be the rational elliptic surface obtained by blowing up at nine branch points of a generic pencil of cubic curves in $\mathbb{C P}^{2}$. Let $E(1)_{p}$ denote the complex surface obtained by performing a logarithmic transformation of multiplicity $p$ on a regular fiber $f$ of $E(1)$. Our main result is the following:

Theorem 1.1. For any integer $p>1$, there exists an infinite family of pairwise nonisotopic symplectic tori representing the primitive homology class $\left[f_{p}\right] \in H_{2}\left(E(1)_{p}\right)$, where $f_{p}$ is the multiple fiber of $E(1)_{p}$.

Even though the tori we construct are not only non-isotopic but also inequivalent in the sense that there is no self-diffeomorphism of the ambient 4-manifold that carries one torus to another, we state the theorem the way we do since our interest in the subject is mainly due to the more general problem of finding non-isotopic symplectic representatives of a homology class, known as the symplectic isotopy problem.

It is well-known that there exists a diffeomorphism between $E(1)_{p}$ and $E(1)$, sending the homology class $\left[f_{p}\right]$ to $[f]$ (see e.g. $[\mathrm{GS} \mid$ ). Hence we immediately obtain the following:

Corollary 1.2. For a suitable choice of a symplectic form on $E(1)$, there exists an infinite family of pairwise non-isotopic symplectic tori representing the primitive homology class $[f] \in H_{2}(E(1))$, where $f$ is the the torus fiber of the elliptic fibration $E(1) \rightarrow \mathbb{C P}^{1}$.

Examples of homologous, non-isotopic, symplectic tori were first constructed in [FS3] and then in [EP1], [EP2] and [V2]. Infinite families of non-isotopic symplectic tori representing divisible classes $n\left[f_{p}\right] \in H_{2}\left(E(1)_{p}\right), n \geq 2$, were constructed in [EP1]. In [EP3] and [V3], symplectic tori representing the same primitive class in symplectic 4-manifolds homeomorphic but not diffeomorphic to $E(1)$ were constructed.

It should be noted that the non-existence of such an infinite family of tori in the rational surfaces $\mathbb{C P}^{2}$ and $\mathbb{C P}^{2} \# \overline{\mathbb{C P}}^{2}$ is proved by Sikorav in [Si] and by Siebert and Tian in

Date: July 28, 2003. Revised on August 28, 2003.

2000 Mathematics Subject Classification. Primary 57R17, 57R57; Secondary 53D35, 57R95.

B.D. Park was partially supported by NSERC and CFI grants. 
[ST], respectively. Moreover, in contrast with Corollary 1.2 which easily generalizes to $\mathbb{C P}^{2} \# n \overline{\mathbb{C P}}^{2}$ for $n \geq 9$, it is conjectured that there is at most one symplectic torus (up to isotopy) representing each homology class in $\mathbb{C P}^{2} \# n \overline{\mathbb{C P}}^{2}$ for $n<9$.

The proof of Theorem 1.1 is spread out over the next four sections. After reviewing the relevant definitions we interpret $E(1)_{p}$ as a link surgery manifold in Section 2 and construct a family of homologous symplectic tori in $E(1)_{p}$ in Section 3 In Section 4 Alexander polynomials of certain braid closures are given and then in Section 5 these polynomials are used to compute the Seiberg-Witten invariants of fiber sums of $E(1)_{p}$ with $E(1)$, along the tori we construct and a regular fiber $f$, respectively, which in turn give the non-isotopy of these tori. In Section 6 we present a direct generalization in the form of Theorem 6.1

In this introduction and elsewhere in the paper by isotopy we mean smooth isotopy and all homology groups have integer coefficients.

\section{LINK SURGERY 4-MANIFOLDS}

In this section, first we review the generalization of the link surgery construction of Fintushel and Stern [FS2] by Vidussi [V1], and then give specific link surgeries that will be used in the following sections.

For an $n$-component link $L \subset S^{3}$, choose an ordered homology basis of simple closed curves $\left\{\left(\alpha_{i}, \beta_{i}\right)\right\}_{i=1}^{n}$ such that the pair $\left(\alpha_{i}, \beta_{i}\right)$ lie in the $i$-th boundary component of the link exterior and the intersection of $\alpha_{i}$ and $\beta_{i}$ is 1 . Let $X_{i}(i=1, \ldots, n)$ be a 4-manifold containing a 2-dimensional torus submanifold $F_{i}$ of self-intersection 0 . Choose a Cartesian product decomposition $F_{i}=C_{1}^{i} \times C_{2}^{i}$, where each $C_{j}^{i} \cong S^{1}(j=1,2)$ is an embedded circle in $X_{i}$.

Definition 2.1. The ordered collection

$$
\mathfrak{D}=\left(\left\{\left(\alpha_{i}, \beta_{i}\right)\right\}_{i=1}^{n},\left\{\left(X_{i}, F_{i}=C_{1}^{i} \times C_{2}^{i}\right)\right\}_{i=1}^{n}\right)
$$

is called a link surgery gluing data for an $n$-component link $L$. We define the link surgery manifold corresponding to $\mathfrak{D}$ to be the closed 4-manifold

$$
L(\mathfrak{D}):=\left[\coprod_{i=1}^{n} X_{i} \backslash \nu F_{i}\right] \underset{F_{i} \times \partial D^{2}=\left(S^{1} \times \alpha_{i}\right) \times \beta_{i}}{\bigcup}\left[S^{1} \times\left(S^{3} \backslash \nu L\right)\right],
$$

where $\nu$ denotes the tubular neighborhoods. Here, the gluing diffeomorphisms between the boundary 3-tori identify the torus $F_{i}=C_{1}^{i} \times C_{2}^{i}$ of $X_{i}$ with $S^{1} \times \alpha_{i}$ factor-wise, and act as the complex conjugation on the last remaining $S^{1}$ factor.

Remark 2.2. Strictly speaking, the diffeomorphism type of the link surgery manifold $L(\mathfrak{D})$ may possibly depend on the chosen trivialization of $\nu F_{i} \cong F_{i} \times D^{2}$ (the framing of $F_{i}$ ). However, we will suppress this dependence in our notation. It is well known (see e.g. [GS]) that the diffeomorphism type of $L(\mathfrak{D})$ is independent of the framing of $F_{i}$ when $\left(X_{i}, F_{i}\right)=(E(1), f)$.

We fix a Cartesian product decomposition of a regular torus fiber $f=C_{1} \times C_{2}$ in the elliptic surface $E(1)$. Also fix $T^{2}:=S^{1} \times S^{1}$.

Definition 2.3. Let $L \subset S^{3}$ be the Hopf link in Figure 1 For the link surgery gluing data

$$
\begin{aligned}
\mathfrak{D}:= & (\{(\mu(A), \lambda(A)),(\mu(B), \lambda(B)-p \mu(B))\}, \\
& \left.\left\{\left(X_{1}, F_{1}=C_{1}^{1} \times C_{2}^{1}\right),\left(T^{2} \times S^{2}, F_{2}=T^{2} \times\{\mathrm{pt}\}\right)\right\}\right),
\end{aligned}
$$

we shall denote $L(\mathfrak{D})$ by $\left(X_{1}\right)_{p}$. Here, $\mu(\cdot)$ and $\lambda(\cdot)$ denote the meridian and the longitude of a knot, respectively. 


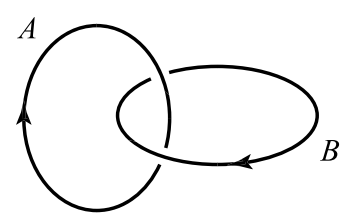

FIGURE 1. Hopf link $L=A \cup B$

In particular, when $\left(X_{1}, F_{1}=C_{1}^{1} \times C_{2}^{1}\right)=\left(E(1), f=C_{1} \times C_{2}\right)$, we denote $L(\mathfrak{D})$ by $E(1)_{p}$. This notation is consistent with the existing literature as there is an obvious diffeomorphism (see diffeomorphism 3.1) in the proof of Lemma 3.1 between our link surgery manifold

$$
L(\mathfrak{D})=[E(1) \backslash \nu f] \cup\left[S^{1} \times\left(S^{3} \backslash L\right)\right] \cup\left[T^{2} \times D^{2}\right]
$$

and the logarithmic transform $E(1)_{p}=[E(1) \backslash \nu f] \cup_{\varphi}\left[T^{2} \times D^{2}\right]$, where the gluing diffeomorphism $\varphi: T^{2} \times \partial D^{2} \rightarrow \partial(\nu f)$ induces the linear map

$$
\varphi_{*}=\left(\begin{array}{rrr}
1 & 0 & 0 \\
0 & 0 & 1 \\
0 & 1 & -p
\end{array}\right)
$$

between the first homology groups with the obvious choice of bases.

Lemma 2.4. If $p \geq 1$, then $E(1)_{p}$ is a symplectic 4-manifold diffeomorphic to $E(1)$. The diffeomorphism type of the logarithmic transform $E(1)_{p}$ does not depend on the choice of the gluing map $\varphi$. The homology class $\left[f_{p}\right] \in H_{2}\left(E(1)_{p}\right)$ is primitive.

Proof. See Theorem 3.3.3, Remark 3.3.5 and Theorem 8.3.11 in [GS].

\section{Family of Homologous Symplectic Tori in $E(1)_{p}$}

Let $T_{C}:=S^{1} \times C \subset\left[S^{1} \times\left(S^{3} \backslash \nu L\right)\right] \subset E(1)_{p}$, where the closed curve $C:=C_{m, p} \subset$ $\left(S^{3} \backslash \nu L\right)$ is given by Figure 2 Note that $C$ could be isotoped to an $(m, m p-1)$ torus knot in $\partial \nu(B) \subset S^{3}$.

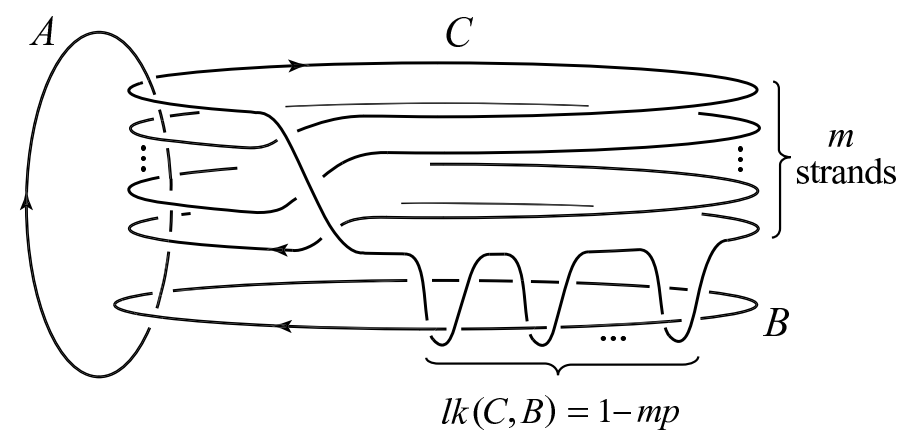

FIGURE 2. 3-component link $L_{m, p}=A \cup B \cup C$ in $S^{3}$

Lemma 3.1. For every pair of integers $p>1$ and $m \geq 1, T_{C}=S^{1} \times C$ is a symplectic submanifold of $E(1)_{p}$ and we have $\left[T_{C}\right]=\left[f_{p}\right]$ in $H_{2}\left(E(1)_{p}\right)$. 
Proof. To determine the homology class of $T_{C}$, note that $[C]=m[\mu(A)]+(1-m p)[\mu(B)]$ in $H_{1}\left(S^{3} \backslash \nu L\right)$. When we glue $\left(T^{2} \times D^{2}\right)$ to $\left(S^{1} \times\left(S^{3} \backslash \nu L\right)\right)$ using the gluing data $\mathfrak{D}$ in 2.1], the homology class $[\mu(A)]-p[\mu(B)]=[\lambda(B)]-p[\mu(B)]$ gets identified with $\left[\{\mathrm{pt}\} \times \partial D^{2}\right] \in H_{1}\left(T^{2} \times D^{2}\right)$, which is trivial. Hence by Künneth's theorem, $\left[T_{C}\right]=$ $\left[S^{1} \times \mu(B)\right]$ in $H_{2}\left(\left(S^{1} \times\left(S^{3} \backslash \nu L\right)\right) \cup\left(T^{2} \times D^{2}\right)\right)$. Finally, note that $\left[S^{1} \times \mu(B)\right]=\left[f_{p}\right]$ and $[f]=\left[S^{1} \times \mu(A)\right]=\left[S^{1} \times \lambda(B)\right]=\left[S^{1} \times p \mu(B)\right]=p\left[f_{p}\right]$ in $H_{2}\left(E(1)_{p}\right)$.

It is easy to see that the link exterior $S^{3} \backslash \nu L$ is diffeomorphic to $S^{1} \times \mathbb{A}$, where $\mathbb{A} \cong$ $S^{1} \times[0,1]$ is an annulus. Hence we may identify the middle piece in the decomposition (2.2) above as the cylinder

$$
\left[S^{1} \times\left(S^{3} \backslash \nu L\right)\right] \cong\left[S^{1} \times\left(S^{1} \times \mathbb{A}\right)\right] \cong T^{3} \times[0,1] .
$$

Since we assume that $p>1$, the logarithmic transform corresponds to the rational blowdown construction of Fintushel and Stern [FS1]. On the other hand, by [Sy], rational blowdown can be done symplectically and the symplectic form needs to be modified only near the collar neighborhood of the boundary lens spaces involved. As a result of this and since $f \simeq p \cdot f_{p}$ remains symplectic in $E(1)_{p}$, we may assume that the symplectic form on $E(1)_{p}$ restricts to

$$
\omega=d x \wedge d y+r d r \wedge d \theta
$$

on the subset $T^{3} \times[\epsilon, 1] \subset\left[S^{1} \times\left(S^{1} \times \mathbb{A}\right)\right]$ for suitable $0<\epsilon<1$, where $x$ and $y$ are the angular coordinates on the first and the second $S^{1}$ factors respectively, and $(r, \theta)$ are the polar coordinates on the annulus $\mathbb{A}$.

We can embed the curve $C$ inside $T^{2} \times[\epsilon, 1] \subset\left(S^{1} \times \mathbb{A}\right)$ such that $C$ is transverse to every annulus of the form, $\{\mathrm{pt}\} \times \mathbb{A}$, and the restriction $\left.d y\right|_{C}$ never vanishes. It follows that $\left.\omega\right|_{T_{C}}=\left.(d x \wedge d y)\right|_{T_{C}} \neq 0$, and consequently $T_{C}$ is a symplectic submanifold of $E(1)_{p}$.

\section{Alexander Polynomials of Certain Braid Closures}

Lemma 4.1. $\Delta_{L_{m, p}}(x, s, t)=$

$$
1-x(s t)^{1-m p}+x t \frac{(x t)^{m-1}-1}{x t-1}\left[1+(s-1) \frac{(s t)^{1-m p}-1}{s t-1}-x(s t)^{1-m p}\right],
$$

where $L_{m, p}$ is the 3-component link in Figure 2 and the variables $x, s$ and $t$ correspond to the components $A, B$ and $C$ respectively.

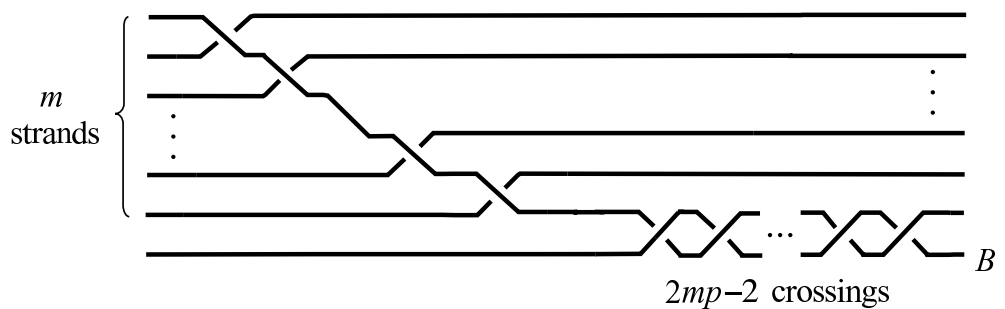

FIGURE 3. $(m+1)$-strand braid whose closure gives $B \cup C$

Proof. This follows from the formula in Theorem 1 of $[\mathrm{Mo}]$ which gives the multivariable Alexander polynomial of a braid closure and its axis in terms of the representation of the braid. We view $A$ as the axis of the $(m+1)$-strand braid in Figure 3 Note that the closure 
of this braid and its axis is $L_{m, p}=A \cup B \cup C$. This statement can also be thought of as a slightly modified special case of Lemma 6.2 in [EP2].

Remark 4.2. Note that the symmetrized Alexander polynomial of $L_{m, p}$ is

$$
\Delta_{L_{m, p}}^{\mathrm{sym}}(x, s, t)=x^{-m / 2} s^{(m p-1) / 2} t^{(m p-m) / 2} \cdot \Delta_{L_{m, p}}(x, s, t) .
$$

\section{NON-ISOTOPY: SEIBERG-WitTEN INVARIANTS}

Our strategy is to show that for fixed choice of $p$ the isotopy types of the tori $\left\{T_{C}\right\}_{m \geq 1}$ can be distinguished by comparing the Seiberg-Witten invariants of the corresponding family of fiber sum 4-manifolds $\left\{E(1)_{p} \#_{T_{C}=f} E(1)\right\}_{m \geq 1}$. Note that there is a canonical framing of a regular fiber $f$ in $E(1)$, coming from the elliptic fibration $E(1) \rightarrow \mathbb{C P}^{1}$.

Lemma 5.1. The fiber sum $E(1)_{p} \#_{T_{C}=f} E(1)$ is diffeomorphic to the link surgery manifold $L_{m, p}\left(\mathfrak{D}^{\prime}\right)$, where

$$
\begin{aligned}
& \mathfrak{D}^{\prime}:=(\{(\mu(A), \lambda(A)),(\mu(B), \lambda(B)-p \mu(B)),(\lambda(C),-\mu(C))\}, \\
&\left.\left\{\left(E(1), f=C_{1} \times C_{2}\right),\left(T^{2} \times S^{2}, F_{2}=S^{1} \times S^{1} \times\{\mathrm{pt}\}\right),\left(E(1), f=C_{1} \times C_{2}\right)\right\}\right) .
\end{aligned}
$$

Proof. It was already observed in [EP2] that the fiber sum construction corresponds to this type of link surgery. (See also [EP3].)

Recall that the Seiberg-Witten invariant $\overline{S W}_{X}$ of a 4-manifold $X$ can be thought of as an element of the group ring of $H_{2}(X)$, i.e. $\overline{S W}_{X} \in \mathbb{Z}\left[H_{2}(X)\right]$. If we write $\overline{S W}_{X}=$ $\sum_{g} a_{g} g$, then we say that $g \in H_{2}(X)$ is a Seiberg-Witten basic class of $X$ if $a_{g} \neq 0$. Since the Seiberg-Witten invariant of a 4-manifold is a diffeomorphism invariant, so are the divisibilities of Seiberg-Witten basic classes. The Seiberg-Witten invariant of the link surgery manifold $L_{m, p}\left(\mathfrak{D}^{\prime}\right)$ is known to be related to the Alexander polynomial $\Delta_{L_{m, p}}$ of the link $L_{m, p}$.

Theorem 5.2. Let $\iota:\left[S^{1} \times\left(S^{3} \backslash \nu L_{m, p}\right)\right] \rightarrow L_{m, p}\left(\mathfrak{D}^{\prime}\right)$ be the inclusion map. Let $\varsigma:=\iota_{*}\left[S^{1} \times \mu(B)\right], \tau:=\iota_{*}\left[S^{1} \times \mu(C)\right] \in H_{2}\left(L_{m, p}\left(\mathfrak{D}^{\prime}\right)\right)$. Then $\varsigma$ and $\tau$ are linearly independent. The Seiberg-Witten invariant of $L_{m, p}\left(\mathfrak{D}^{\prime}\right)$ is given by

$$
\begin{aligned}
\overline{S W}_{L_{m, p}\left(\mathfrak{D}^{\prime}\right)}= & \varsigma^{-m p+2+2 p} \tau^{-m p^{2}+m p} . \\
& {\left[\left(\varsigma^{2 m p-2 p-4}+\cdots+\varsigma^{2}+1\right) \cdot\right.} \\
& \left(\varsigma^{2 m p-2 p} \tau^{2 m^{2} p-2 m p}+\cdots+\varsigma^{2 p} \tau^{2 m p}+1\right) \\
& +\tau^{2}\left(\varsigma^{2 m p-4} \tau^{2 m p-4}+\cdots+\varsigma^{2} \tau^{2}+1\right) \cdot \\
& \left.\left(\varsigma^{2 m p-4 p} \tau^{2 m^{2} p-4 m p}+\cdots+\varsigma^{2 p} \tau^{2 m p}+1\right)\right] .
\end{aligned}
$$

Proof. Let $N:=\left(S^{3} \backslash \nu L_{m, p}\right)$, and let $Z:=\left(T^{2} \times D^{2}\right)$. Recall from [Pa] that we have $\overline{S W}_{E(1) \backslash \nu f}=1$, and also

$$
\overline{S W}_{\left(T^{2} \times D^{2}\right)}^{ \pm}=\frac{1}{\left[T^{2} \times\{\mathrm{pt}\}\right]^{-1}-\left[T^{2} \times\{\mathrm{pt}\}\right]} .
$$

From the gluing formulas in $[\mathrm{Pa} \mid$ and $[\mathrm{Ta} \mid$, we may conclude that

$$
\begin{aligned}
\overline{S W}_{L_{m, p}\left(\mathcal{D}^{\prime}\right)} & =\overline{S W}_{E(1) \backslash \nu f} \cdot{\overline{S W_{E(1) \backslash \nu f}}} \cdot \overline{S W}_{\left(T^{2} \times D^{2}\right)}^{ \pm} \cdot \Delta_{L_{m, p}}^{\mathrm{sym}}\left(\xi^{2}, \varsigma^{2}, \tau^{2}\right) \\
& =\frac{1}{\left[T^{2} \times\{\mathrm{pt}\}\right]^{-1}-\left[T^{2} \times\{\mathrm{pt}\}\right]} \cdot \Delta_{L_{m, p}}^{\mathrm{sym}}\left(\xi^{2}, \varsigma^{2}, \tau^{2}\right),
\end{aligned}
$$


where $\xi:=\iota_{*}\left[S^{1} \times \mu(A)\right]$.

First of all, $[\lambda(B)]=[\mu(A)]+(1-m p)[\mu(C)] \in H_{1}(N)$. On the other hand, according to the gluing data $\mathfrak{D}^{\prime}, \lambda(B)-p \mu(B)$ is identified with $\{\mathrm{pt}\} \times \partial D^{2} \in Z$, therefore $[\lambda(B)]=$ $p[\mu(B)]$ in $L_{m, p}\left(\mathfrak{D}^{\prime}\right)$ and consequently

$$
\xi=\varsigma^{p} \tau^{m p-1} .
$$

Also note that $T^{2} \in T^{2} \times S^{2}$ and $S^{1} \times \mu(B)$ are identified by the gluing data $\mathfrak{D}^{\prime}$, hence

$$
\left[T^{2} \times\{p t\}\right]=\varsigma \in H_{2}\left(L_{m, p}\left(\mathfrak{D}^{\prime}\right)\right) .
$$

From Lemma4.1 and Remark 4.2 we have

$$
\begin{aligned}
& \Delta_{L_{m, p}}^{\mathrm{sym}}\left(\left(\varsigma^{p} \tau^{m p-1}\right)^{2}, \varsigma^{2}, \tau^{2}\right)=\varsigma^{-1} \tau^{-m^{2} p+m p} \cdot\left[1-\varsigma^{-2 m p+2 p+2}\right. \\
+ & \left.\varsigma^{2 p} \tau^{2 m p} \frac{\left(\varsigma^{2 p} \tau^{2 m p}\right)^{m-1}-1}{\varsigma^{2 p} \tau^{2 m p}-1}\left(1+\left(\varsigma^{2}-1\right) \frac{\left(\varsigma^{2} \tau^{2}\right)^{1-m p}-1}{\varsigma^{2} \tau^{2}-1}-\varsigma^{-2 m p+2 p+2}\right)\right] .
\end{aligned}
$$

After putting Equations (5.3), (5.4), (5.5) and (5.6) together and simplifying the resulting expression, we get (5.2).

Next we show that $\varsigma$ and $\tau$ are linearly independent elements of $H_{2}\left(L_{m, p}\left(\mathfrak{D}^{\prime}\right)\right)$. A Mayer-Vietoris argument, combined with Freedman's classification theorem (see, for example, $|\overline{\mathrm{FQ}}|)$, shows that $L_{m, p}\left(\mathfrak{D}^{\prime}\right)$ is homeomorphic to $E(2)$.

Consider the composition of homomorphisms

$$
H_{1}(N) \longrightarrow H_{2}\left(S^{1} \times N\right) \stackrel{\iota_{*}}{\longrightarrow} H_{2}\left(L_{m, p}\left(\mathfrak{D}^{\prime}\right)\right),
$$

where the first map is a part of the Künneth isomorphism

$$
H_{1}(N) \oplus H_{2}(N) \stackrel{\cong}{\longrightarrow} H_{2}\left(S^{1} \times N\right) .
$$

Note that $H_{2}(N) \cong \mathbb{Z} \oplus \mathbb{Z}$, as is easily seen from the long exact sequence of the pair $(N, \partial N)$ as follows.

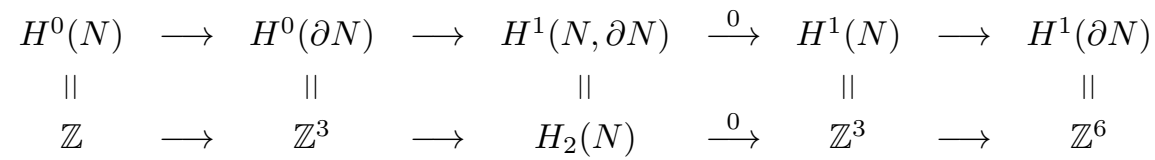

Note that the first map sends the generator $1 \in \mathbb{Z}$ to the diagonal element $(1,1,1) \in \mathbb{Z}^{3}$, while the last map is injective. We have also used the Lefschetz duality theorem (for manifolds with boundary) to identify $H_{2}(N) \cong H^{1}(N, \partial N)$.

Next consider the long exact sequence of the pair $\left(L_{m, p}\left(\mathfrak{D}^{\prime}\right), S^{1} \times N\right)$ :

$$
0=H_{3}\left(L_{m, p}\left(\mathfrak{D}^{\prime}\right)\right) \longrightarrow H_{3}\left(L_{m, p}\left(\mathfrak{D}^{\prime}\right), S^{1} \times N\right) \longrightarrow H_{2}\left(S^{1} \times N\right) \stackrel{\iota_{*}}{\longrightarrow} H_{2}\left(L_{m, p}\left(\mathfrak{D}^{\prime}\right)\right)
$$

The kernel of the last map $\iota_{*}$ is isomorphic to $H_{3}\left(L_{m, p}\left(\mathfrak{D}^{\prime}\right), S^{1} \times N\right)$. By Lefschetz duality theorem (for relative manifolds), $H_{3}\left(L_{m, p}\left(\mathfrak{D}^{\prime}\right), S^{1} \times N\right)$ is in turn isomorphic to

$$
H^{1}\left(L_{m, p}\left(\mathfrak{D}^{\prime}\right) \backslash\left(S^{1} \times N\right)\right) \cong H^{1}(E(1) \backslash \nu f) \oplus H^{1}(E(1) \backslash \nu f) \oplus H^{1}(Z) .
$$

Since we have $H^{1}(E(1) \backslash \nu f)=0$ and

$$
H^{1}(Z)=H^{1}\left(T^{2} \times D^{2}\right) \cong \mathbb{Z} \oplus \mathbb{Z},
$$

the kernel of $\iota_{*}$ is isomorphic to $\mathbb{Z} \oplus \mathbb{Z}$. 
Finally we observe that only one $\mathbb{Z}$ summand of 5.9 lies in the kernel of the composition (5.7). The other $\mathbb{Z}$ summand belongs to the kernel of

$$
H_{2}(N) \longrightarrow H_{2}\left(S^{1} \times N\right) \stackrel{\iota_{*}}{\longrightarrow} H_{2}\left(L_{m, p}\left(\mathfrak{D}^{\prime}\right)\right),
$$

where the first map is the second part of the Künneth isomorphism (5.8). We have thus shown that the kernel of the composition (5.7) is of rank one. It follows immediately that $\varsigma$ and $\tau$ are linearly independent, since we have already showed that $\xi=\varsigma^{p} \tau^{m p-1}$. A more detailed analysis shows that $\{\varsigma, \tau\}$ can be extended to a basis of $H_{2}\left(L_{m, p}\left(\mathfrak{D}^{\prime}\right)\right)$, which we shall omit. (Also see the proof of Proposition 3.2 in [MT] for a similar argument.)

Corollary 5.3. The number of Seiberg-Witten basic classes of $L_{m, p}(\mathfrak{D})$ is

$$
2 p m^{2}-2(p+1) m+1 .
$$

Proof. First of all, the summands in Equation 5.2 have no common terms, i.e. terms with matching powers for $\varsigma$ and $\tau$, because the general term in the first summand is $\left(\varsigma^{2}\right)^{k}\left(\varsigma^{2 p} \tau^{2 m p}\right)^{l}$, where $0 \leq k \leq m p-p-2$ and $0 \leq l \leq m-1$, the general term in the second summand is $\tau^{2}\left(\varsigma^{2} \tau^{2}\right)^{i}\left(\varsigma^{2} p \tau^{2 m p}\right)^{j}$, where $0 \leq i \leq m p-2$ and $0 \leq j \leq m-2$, and in order for two such terms to have matching powers, for even only $\tau, 2 \operatorname{lmp}$ should be the same as $2+2 i+2 j m p$ which would imply $i \equiv-1(\bmod m p)$ contradicting $0 \leq i \leq m p-2$. Therefore, to get the number of basic classes, we simply add the number of terms in each of these summands.

Corollary 5.4. There is no self-diffeomorphism of $E(1)_{p}$ that maps one element of the family of tori $\left\{T_{C}\right\}_{m \geq 1}$ to another. In particular, these tori are pairwise non-isotopic.

Proof. Since the Seiberg-Witten invariant is a diffeomorphism invariant, so are the number of basic classes. On the other hand, by Corollary 5.3, the number of basic classes of $L_{m, p}\left(\mathfrak{D}^{\prime}\right)$ is $2 p m^{2}-2(p+1) m+1$ and for fixed $p \geq 2$ this polynomial of $m$ is obviously increasing when $m \geq 1$. Hence $L_{m, p}\left(\mathfrak{D}^{\prime}\right)$ is diffeomorphic to $L_{m^{\prime}, p}\left(\mathfrak{D}^{\prime}\right)$ if and only if $m=m^{\prime}$ proving that the tori in $\left\{T_{C}\right\}_{m \geq 1}$ are different up to isotopy and in fact even up to self-diffeomorphisms of $E(1)_{p}$.

This concludes the proof of Theorem 1.1

\section{Generalization to Other Symplectic 4-Manifolds}

We can easily extend Theorem 1.1 to $E(n)_{p}(n \geq 2)$ and more generally to $X_{p}$, where $X$ is a symplectic 4-manifold satisfying certain topological conditions as in [EP1].

Theorem 6.1. Assume that $F$ is a symplectic 2-torus in a symplectic 4-manifold $X$. Suppose that $[F] \in H_{2}(X)$ is primitive, $[F] \cdot[F]=0$, and that $F$ lies in a fishtail neighborhood. If $b_{2}^{+}(X)=1$, then we also assume that $\overline{S W}_{X \backslash \nu F} \neq 0$ and is a finite sum. Then there exists an infinite family of pairwise non-isotopic symplectic tori in $X_{p}$ representing the homology class $\left[F_{p}\right] \in H_{2}\left(X_{p}\right)$ for any integer $p>1$. These tori are inequivalent under self-diffeomorphisms of $X_{p}$.

Proof. It was shown in [FS1] and [Sy] that $X_{p}$ possesses a canonical symplectic form coming from the symplectic form on $X$. The rest of the proof goes the same way as before.

Acknowledgments. We would like to thank Ronald Fintushel and Stefano Vidussi for their encouragement and helpful comments on this and other works of ours. 


\section{REFERENCES}

[EP1] T. Etgü and B.D. Park: Non-isotopic symplectic tori in the same homology class, preprint. Available at arXiv:math.GT/0212356

[EP2] T. Etgü and B.D. Park: Homologous non-isotopic symplectic tori in a $K 3$-surface, preprint. Available at arXiv:math.GT/0305201

[EP3] T. Etgü and B.D. Park: Homologous non-isotopic symplectic tori in homotopy rational elliptic surfaces, preprint. Available at arXiv:math.GT/0307029

[FS1] R. Fintushel and R.J. Stern: Rational blowdown of smooth 4-manifolds, J. Differential Geom. 46 (1997), 181-235.

[FS2] R. Fintushel and R.J. Stern: Knots, links and 4-manifolds, Invent. Math. 134 (1998), 363-400.

[FS3] R. Fintushel and R.J. Stern: Symplectic surfaces in a fixed homology class, J. Differential Geom. 52 (1999), 203-222.

[FQ] M.H. Freedman and F. Quinn: Topology of 4-Manifolds, Princeton University Press, 1990.

[GS] R.E. Gompf and A.I. Stipsicz: 4-Manifolds and Kirby Calculus, Graduate Studies in Mathematics 20, Amer. Math. Soc., 1999.

[MT] C.T. McMullen and C.H. Taubes: 4-manifolds with inequivalent symplectic forms and 3-manifolds with inequivalent fibrations, Math. Res. Lett. 6 (1999), 681-696.

[Mo] H.R. Morton: The multivariable Alexander polynomial for a closed braid, in Low-dimensional Topology, ed. Hanna Nencka, Contemporary Mathematics 233, Amer. Math. Soc. (1999), 167-172. Also available at arXiv:math.GT/9803138

[Pa] B.D. Park: A gluing formula for the Seiberg-Witten invariant along $T^{3}$, Michigan Math. J. 50 (2002), 593-611.

[ST] B. Siebert and G. Tian: On the holomorphicity of genus two Lefschetz fibrations, preprint. Available at arXiv:math.SG/0305343

[Si] J.-C. Sikorav: The gluing construction for normally generic $J$-holomorphic curves, in Symplectic and Contact Topology: Interactions and Perspectives (Toronto, ON/Montréal, QC, 2001), 175-198, Fields Inst. Commun. 35, Amer. Math. Soc., 2003. Also available at arXiv:math.SG/0102004

[Sy] M. Symington: Symplectic rational blowdowns, J. Differential Geom. 50 (1998), 505-518.

[Ta] C.H. Taubes: The Seiberg-Witten invariants and 4-manifolds with essential tori, Geom. Topol. 5 (2001), 441-519.

[V1] S. Vidussi: Smooth structure of some symplectic surfaces, Michigan Math. J. 49 (2001), 325-330.

[V2] S. Vidussi: Nonisotopic symplectic tori in the fiber class of elliptic surfaces, preprint. Available at http://www.math.ksu.edu/ vidussi/

[V3] S. Vidussi: Symplectic tori in homotopy $E(1)$ 's, preprint. Available at http://www.math.ksu.edu/ vidussi/

Department of Mathematics and Statistics, McMaster University, Hamilton, Ontario, L8S 4K1, CANADA

E-mail address: etgut @math.mcmaster.ca

Department of Pure Mathematics, University of Waterloo, Waterloo, Ontario, N2L 3G1, CANADA

E-mail address: bdpark@math. uwaterloo.ca 